\title{
A clinical metabolomics-based biomarker signature as an approach for early diagnosis of gastric cardia adenocarcinoma
}

\author{
YUANFANG SUN $^{1 *}$, SHASHA LI $^{2 *}$, JIN LI $^{3}$, XUE XIAO $^{4}$, ZHAOLAI HUA $^{5}$, XI WANG $^{3}$ and SHIKAI YAN ${ }^{1}$ \\ ${ }^{1}$ School of Pharmacy, Shanghai Jiao Tong University, Shanghai 200240; ${ }^{2}$ The Second Clinical College of \\ Guangzhou University of Chinese Medicine, Guangzhou, Guangdong 510006; ${ }^{3}$ Department of Oncology, The 903rd \\ Hospital of PLA, Hangzhou, Zhejiang 310013; ${ }^{4}$ Institute of Traditional Chinese Medicine, Guangdong \\ Pharmaceutical University, Guangzhou, Guangdong 510006; ${ }^{5}$ People's Hospital of Yangzhong, \\ Yangzhong, Jiangsu 212200, P.R. China
}

Received May 14, 2019; Accepted October 10, 2019

DOI: $10.3892 / \mathrm{ol} .2019 .11173$

\begin{abstract}
Gastric cardia adenocarcinoma (GCA) has a high mortality rate worldwide; however, current early diagnostic methods lack efficacy. Therefore, the aim of the present study was to identify potential biomarkers for the early diagnosis of GCA. Global metabolic profiles were obtained from plasma samples collected from 21 patients with GCA and 48 healthy controls using ultra-performance liquid chromatography/quadrupole-time-of-flight mass spectrometry. The orthogonal partial least squares discrimination analysis model was applied to distinguish patients with GCA from healthy controls and to identify potential biomarkers. Metabolic pathway analysis was performed using MetaboAnalyst (version 4.0) and revealed that 'glycerophospholipid metabolism', 'linoleic acid metabolism', 'fatty acid biosynthesis' and 'primary bile acid biosynthesis' were significantly associated with GCA. In addition, an early diagnostic model for GCA was established based on the relative levels of four key biomarkers, including
\end{abstract}

Correspondence to: Dr Xi Wang, Department of Oncology, The 903rd Hospital of PLA, 40 Jichang Road, Hangzhou, Zhejiang 310013, P.R. China

E-mail: d.wangxi@hotmail.com

Dr Shikai Yan, School of Pharmacy, Shanghai Jiao Tong University, 800 Dongchuan Road, Shanghai 200240, P.R. China

E-mail: shkyan@126.com

*Contributed equally

Abbreviations: GCA, gastric cardia adenocarcinoma; UPLC/Q-TOF MS, ultra-performance liquid chromatography/quadrupole-time-offlight mass spectrometry; OPLS-DA, orthogonal partial least squares discrimination analysis; ROC, receiver operating characteristic

Key words: gastric cardia adenocarcinoma, early diagnosis, metabolomics, biomarkers, ultra-performance liquidchromatography/ quadrupole-time-of-flight mass spectrometry phosphorylcholine, glycocholic acid, L-acetylcarnitine and arachidonic acid. The area under the receiver operating characteristic curve revealed that the diagnostic model had a sensitivity and specificity of 0.977 and 0.952 , respectively. The present study demonstrated that metabolomics may aid the identification of the mechanisms underlying the pathogenesis of GCA. In addition, the proposed diagnostic method may serve as a promising approach for the early diagnosis of GCA.

\section{Introduction}

Gastric cardia adenocarcinoma (GCA) is characterized by short disease duration and poor prognosis (1). GCA has a high incidence rate ( $\sim 3.3$ new cases per 100,000 per year) worldwide (2), and is much higher in China, with 49.59 new cases per 100,000 per year (3). GCA presents with non-specific symptoms at the early stages of the disease, and the majority of patients are diagnosed at an advanced stage; consequently, patients with GCA have a low survival rate $(4,5)$. Previous studies have revealed that early diagnosis and treatment of GCA improves patient outcomes and results in a more favorable prognosis $(6,7)$. The currently available methods for the early diagnosis of GCA include barium X-ray radiography $(8,9)$, endoscopy $(10,11)$, abdominal ultrasound (12), spiral computed tomography $(13,14)$ and proton nuclear magnetic resonance $(15,16)$. However, these methods are invasive and not cost-effective (17). Therefore, there is a requirement for a non-invasive, cost-effective and efficient method to diagnose early GCA and improve patient prognosis.

Systems biology and high-throughput screening methods have revealed that the incidence of GCA is associated with mutations in tumor protein P53 $(18,19)$ and the vitamin D receptor (20), as well as polymorphisms in the Fas ligand gene (21) and thymidylate synthase (22). In addition, proteins such as cancer antigen 125 and minichromosome maintenance 5 serve as potential biomarkers for the early diagnosis of GCA $(23,24)$. However, the aforementioned genes and proteins lack specificity and sensitivity, and previous studies have revealed that a single biomarker is unlikely to accurately diagnose GCA $(23,25)$. Laser capture microdissection-based 
proteome analysis on human GCA tissues revealed that 23 proteins are abnormally expressed in patients with GCA compared with healthy controls (26). Metabolomics has been widely applied for the diagnosis for several types of cancer, including colorectal (27,28), liver (29), pancreatic $(30,31)$ and gastric $(32,33)$ cancer. However, a limited number of studies have investigated a metabolomics-based approach for the diagnosis of GCA $(34,35)$. Cai et al (34) have suggested that the metabolites and proteins associated with glycolysis may serve as potential biomarkers for the diagnosis of GCA; however, an established diagnostic model has not been reported.

The present study aims to identify potential biomarkers for the early detection of GCA. The ultra-performance liquid chromatography/quadrupole-time-of-flight mass spectrometry (UPLC/Q-TOF MS) was utilized to establish the metabolic fingerprints of patients with GCA and healthy controls, and the specific biomarkers associated with GCA were screened to develop an early diagnostic model. Furthermore, metabolic pathway analysis was performed to investigate the pathways associated with the pathogenesis of GCA.

\section{Materials and methods}

Chemicals. Isopropanol [high-performance liquid chromatography (HPLC)-grade], acetonitrile (HPLC-grade), methanol (HPLC-grade) and formic acid (98\%) were purchased from J.T. Baker; Avantor, Inc. The internal standard L-2 chlorophenylalanine was obtained from Ark Pharm, Inc. and ultrapure water was purchased from EMD Millipore.

Clinical samples. The present study was approved by the Ethics Committee of the People's Hospital of Yangzhong City (Yangzhong, China) and all participants provided written informed consent. Plasma samples were obtained from 21 patients with GCA (14 males and 7 females; mean age, 66 years; age range, $60-80$ years) and 48 healthy volunteers (14 males and 34 females; mean age, 52.15 years; age range, 40-68 years) at the People's Hospital of Yangzhong City between July and December 2015. Patients with GCA were diagnosed by gastroscopy and had not received chemotherapy or other therapy, including surgery and radiotherapy. A mucosal biopsy was performed to classify patients according to the Tumor-Node-Metastasis (TNM) Classification of Malignant Tumors (36). The clinical information of the participants is summarized in Table I.

A total of $5 \mathrm{ml}$ peripheral venous blood was obtained from the participants and collected in anticoagulation tubes containing heparin sodium. The blood was centrifuged at $670 \mathrm{x} \mathrm{g}$ for $15 \mathrm{~min}$ at $4^{\circ} \mathrm{C}$, and the supernatant was collected and stored at $-80^{\circ} \mathrm{C}$ until further analysis.

Sample preparation. Plasma samples were thawed on ice prior to analysis. A total of $100 \mu \mathrm{l}$ plasma was added to $300 \mu \mathrm{l}$ methanol and acetonitrile mixture (1:1) containing $5 \mu \mathrm{g} / \mathrm{ml}$ L-2 chlorophenylalanine internal standard, which was used to check the integrity of the automated integration and to assess instrument performance throughout the batch analysis. The mixture was vortexed for $30 \mathrm{sec}$ and maintained at $4^{\circ} \mathrm{C}$ for $1 \mathrm{~h}$, vortexed again for $30 \mathrm{sec}$ and maintained at $4^{\circ} \mathrm{C}$ for $3 \mathrm{~h}$. The mixture was vortexed and centrifuged at $37,730 \mathrm{xg}$ for $10 \mathrm{~min}$ at $4^{\circ} \mathrm{C}$. Subsequently, $300 \mu \mathrm{l}$ of the supernatant was centrifuged at $37,730 \mathrm{x} \mathrm{g}$ for $10 \mathrm{~min}$ at $4^{\circ} \mathrm{C}$, and $200 \mu \mathrm{l}$ supernatant was decanted into a vial with an inner cannula.

To monitor the robustness of sample preparation and the stability of instrument analysis, a quality control (QC) sample was prepared by pooling $10 \mu \mathrm{l}$ plasma from all samples.

UPLC-Q/TOF MS analysis. The UPLC analysis was performed in a Waters Acquity ${ }^{\mathrm{TM}}$ Ultra-performance LC system coupled with a Waters Micromass ${ }^{\text {TM }}$ Q/TOF MS (Waters Corporation). The chromatographic separation was performed on an ACQUITY BEH-C18 column (2.1x100 mm; $1.7 \mu \mathrm{m}$; Waters Corporation). The mobile phase is composed of (A) $0.1 \%$ formic acid and (B) isopropanol, acetonitrile and methanol [20:40:40 (v/v)] in 0.1\% formic acid, and the gradient was used as follows: $98-50 \%$ (A) and 2-50\% (B) for 0-3.5 min; $50-0 \%(\mathrm{~A})$ and $50-100 \%$ (B) for $3.5-20 \mathrm{~min}$. The flow rate was $0.4 \mathrm{ml} / \mathrm{min}$. The temperature of the autosampler and the chromatographic column was maintained at 4 and $50^{\circ} \mathrm{C}$, respectively. A total of $5 \mu \mathrm{l}$ sample solution was injected per run. The MS scan ranged from $50-1,000 \mathrm{~m} / \mathrm{z}$ in the positive and negative ion mode. The cone and capillary voltages were set at $35 \mathrm{~V}$ and $3.0 \mathrm{kV}$ for positive ionization mode, and $35 \mathrm{~V}$ and $2.8 \mathrm{kV}$ for negative ionization mode. The collision energy was set at $4 \mathrm{eV}$. A desolvation gas flow rate of $600 \mathrm{l} / \mathrm{h}$ at $115^{\circ} \mathrm{C}$ was used. The data acquisition rate was set to $0.3 \mathrm{spec} / \mathrm{sec}$. Data acquisition was performed using Waters MassLynx software (version 4.1; Waters Corporation).

Data processing. Prior to statistical analysis, raw data were imported into the Progenesis QI software package (version 2.0; Waters Corporation) for data standardization. The orthogonal partial least squares discriminant analysis (OPLS-DA) model was performed using SIMCA software (version 14.1; Umetrics Ltd.). Potential biomarkers were identified from loading plots constructed following analysis with OPLS-DA, and the biomarkers were selected based on the variable importance in the projection (VIP) value. The model parameters of R2Y(cum) is used to estimate the 'goodness of fit' of the model, and Q2 (cum) estimates the ability of prediction. The S-plot is used to visualize both the covariance and the correlation structure between the $\mathrm{X}$-variables and the predictive score $t[1]$. Thus, the S-plot is a scatter plot of the p[1] vs. p(corr) [1] vectors of the predictive component. A response permutation test with 200 iterations was performed to assess the risk that the current OPLS-DA model is spurious. The structure of the differential metabolites was identified using the Human Metabolome Database (HMDB; version 4.0; www.hmdb. ca/spectra/ms/search) and The LIPID MAPS Lipidomics Gateway (updated March 9th, 2016; www.lipidmaps.org) based on secondary mass spectrometry information.

Statistical analysis. Statistical analyses were performed using SPSS software (version 19.0; IBM Crop.). Student's t-test was performed to compare the differences in metabolite levels between healthy subjects and patients with GCA. $\mathrm{P}<0.05$ was considered to indicate a statistically significant difference. The diagnostic abilities of the biomarkers were assessed by systematic cluster analysis, receiver operating characteristic 
Table I. Clinical information of the enrolled subjects.

\begin{tabular}{lcc}
\hline Characteristics & Healthy & GCA \\
\hline Sex (male/female) & $14 / 34$ & $14 / 7$ \\
Age, years & $52.15 \pm 6.96$ & $66.00 \pm 8.48$ \\
TNM ${ }^{\text {a }}$ stage & & \\
IA & - & 2 \\
IB & - & 1 \\
IIA & - & 1 \\
IIIA & - & 7 \\
IIIC & - & 9 \\
IV & - & 1 \\
\hline
\end{tabular}

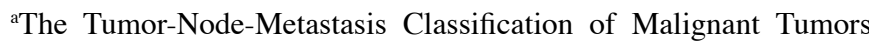
(TNM) is a globally recognized standard for classifying the extent of spread of cancer. It is a classification system of the anatomical extent of tumor cancers. $T$ describes the size of the original (primary) tumor and whether it has invaded nearby tissue, $\mathrm{N}$ describes nearby (regional) lymph nodes that are involved, $\mathrm{M}$ describes distant metastasis (spread of cancer from one part of the body to another) (67). GCA, gastric cardia adenocarcinoma.

(ROC) curves and binary logistic regression. In addition, the pathway analysis module of MetaboAnalyst software (version 4.0; www.metaboanalyst.ca) (37) was used to analyze significant metabolic pathways, which has pathway impact value of no less than 0.1 and $-\log (\mathrm{p})$ value of no less than 2 .

\section{Results}

UPLC-Q/TOF MS analysis of plasma samples. A total of 69 samples were analyzed in a random order, and QCs were inserted into the analysis sequence to monitor and correct changes in the instrument response. To identify and remove probable characteristic peaks caused by source contaminants, test tube components or solvent impurities, blank samples were inserted every 10 runs. Analyses were performed in the positive and negative ion mode (Fig. 1).

The method validation was performed based on precision and stability testing, which permitted $<10 \%$ relative standard deviation. The results demonstrated the stability of the instrument and the samples (Tables SI-SIV).

Multivariate statistical analysis. In the present study, multivariate statistical analysis and pattern recognition methods, principal component analysis (PCA) and OPLS-DA, were used to analyze the metabolomics data. PCA resulted in poor distinction between patients and healthy subjects (Fig. S1). The OPLS-DA model was subsequently applied to the metabolomics data to eliminate interference from various non-experimental factors, including sex and age. The model exhibited a trend for distinguishing between patients with GCA and controls (Fig. 2A and B). The model parameters of R2Y(cum) and Q2 (cum) were 89.6 and $62.1 \%$ in the negative pattern and 73.1 and $55.4 \%$ in positive pattern, respectively, which indicated that the model had a good fit and predictive ability.
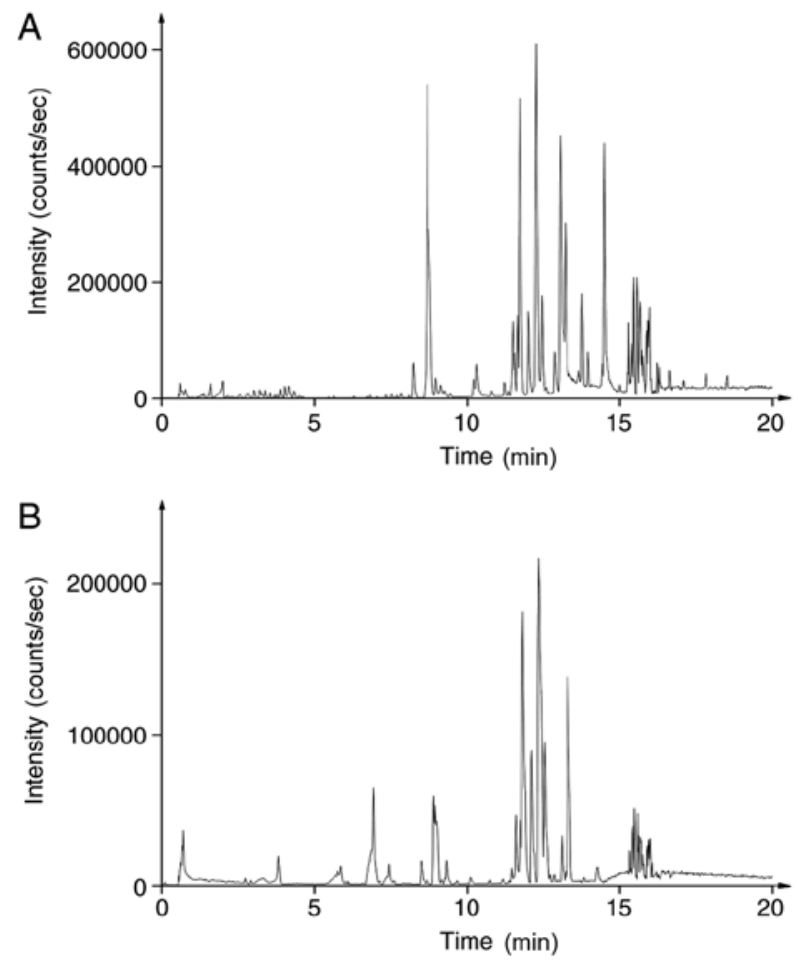

Figure 1 . The base peak chromatograms of the quality control samples in the (A) positive and (B) negative ion modes.

Identification of potential biomarkers. A total of 27 differential metabolites based on VIP $>1$ with S-plot (Fig. 2C and D) and $\mathrm{P}<0.05$ (Table II), including L-acetylcarnitine, arachidonic acid, phosphorylcholine and glycocholic acid, were identified and used to establish the OPLS-DA model (Fig. 2E). This model was able to clearly distinguish patients with GCA from healthy subjects (99.7 and $99.2 \%$ for R2Y and Q2, respectively), and compared with Fig. 2A and B, the clustering effect was improved. Following the 200 iterations of the permutation test, the results revealed that the OPLS-DA model did not result in overfitting as the left replacement R2 and Q2 values were lower than the original point on the right, and the Q2 intercept was $<0$ (Fig. 2F). Therefore, the identified 27 differential metabolites may serve as potential biomarkers for the diagnosis of GCA.

Cluster analysis revealed that the 27 potential biomarkers could be divided into fourcategories: i) Phospholipids (including phosphorylcholine); ii) cholic acid (including glycocholic acid); iii) unsaturated fatty acids (including arachidonic acid); and iv) amino acids (including L-acetylcarnitine; Fig. 3A). ROC curve analysis was performed for the candidate biomarkers, and the area under the curve (AUC), sensitivity and specificity were determined (Tables III and SV). Phosphorylcholine, glycocholic acid, arachidonic acid and L-acetylcarnitine had the largest AUC values $(0.913,0.808,0.887$ and 0.803 , respectively), and were thus selected for binary logistic regression to establish a joint index and plot ROC curves (Fig. 3F). The AUC value of this four biomarker model was 0.990 for the discrimination between patients with GCA and healthy subjects. In addition, the sensitivity and specificity of the model for GCA diagnosis were 97.7 and $95.2 \%$, respectively. Compared with single biomarker models using phosphorylcholine, glycocholic 

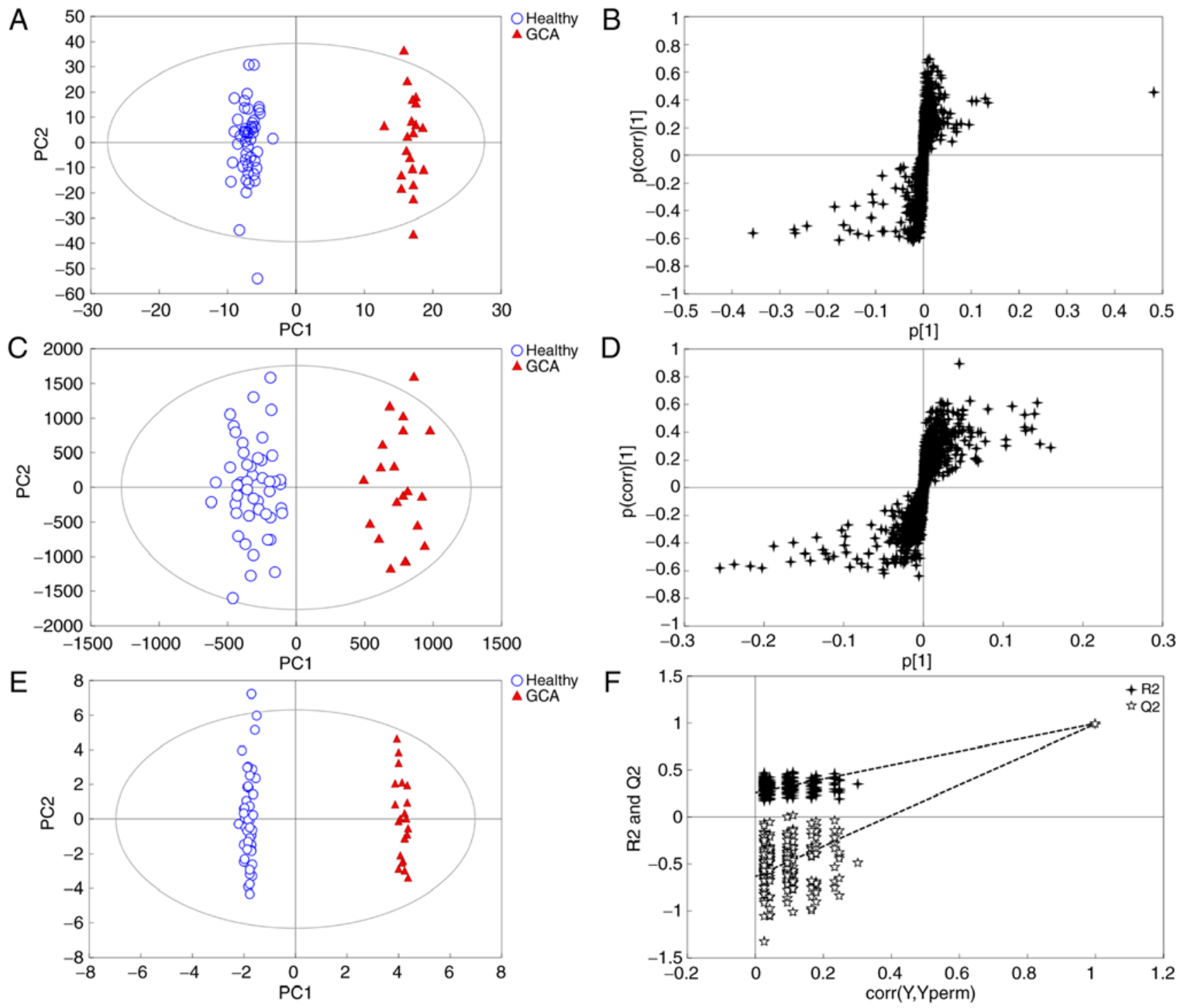

Figure 2. Multivariate statistical analysis for GCA (blue circles) and healthy controls (red triangles). (A) OPLS-DA score plot with all variables Pareto scaled using the metabolomics data in the positive ion modes. (B) S-plot of all compounds that were detected in the positive ion modes. (C) OPLS-DA score plot with all variables Pareto scaled using the metabolomics data in the negative ion modes. (D) S-plot of all compounds that were detected in the negative ion modes. (E) OPLS-DA score plot based on 27 differential metabolites of GCA. (F) Permutation test following 200 iterations. The vertical axis corresponds to R2 and Q2 values of each model. R2 was the estimate of 'goodness of fit' of the model, and Q2 is the estimate of the ability of prediction. The horizontal axis corresponds to the correlation coefficient between the original Y and the permuted Y. GCA, gastric cardia adenocarcinoma; OPLS-DA, orthogonal partial least squares discrimination analysis.

acid, arachidonic acid and L-acetylcarnitine individually, a model comprising the aforementioned four metabolites exhibited improved AUC values, sensitivity and specificity (Fig. 3B-F).

Metabolic pathway analysis. To gain a deeper understanding of the biological significance of the potential biomarkers identified in the present study, MetaboAnalyst software (version 4.0) was used for metabolic pathway analysis. The results demonstrated that 'glycerophospholipid metabolism', 'linoleic acid metabolism', 'primary bile acid biosynthesis', 'fatty acid synthesis', 'arachidonic acid metabolism', 'seleno amino acid metabolism' and 'aminoacyl-tRNA biosynthesis' pathways were closely associated with GCA (Figs. 4 and 5). These pathways are mainly associated with energy metabolism, inflammatory reactions and immune responses.

\section{Discussion}

The lack of an effective diagnostic model is the major cause contributing to the high mortality of GCA (38). To date, a limited number of biomarkers for GCA diagnosis have been identified $(23,25,39)$. The present study identified 27 biomarkers and established a combined diagnostic model comprising phosphorylcholine, glycocholic acid, arachidonic acid and L-acetylcarnitine for the early diagnosis of GCA. Metabolic pathway enrichment analysis was performed based on all 27 biomarkers identified in the present study. The results revealed that disruptions in glycerophospholipid and linoleic acid metabolism and fatty acid and primary bile acid biosynthesis were significantly associated with the development of GCA. These metabolic disturbances may help understand the underlying pathogenesis of the disease. 


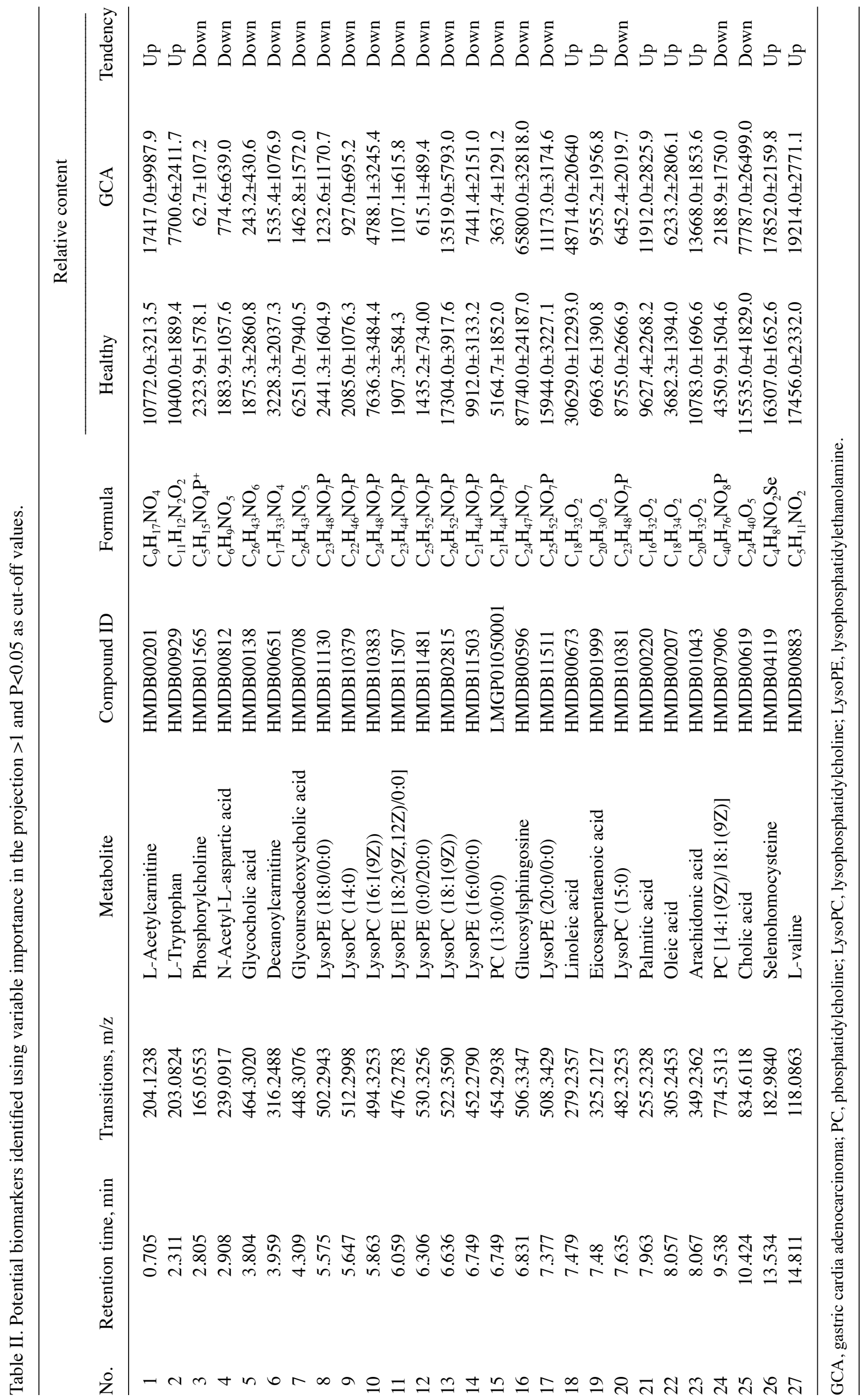


Table III. Receiver operating characteristic curve analysis of the four biomarkers (phosphorylcholine, L-acetylcarnitine, arachidonic acid and glycocholic acid) and the combined model.

$95 \% \mathrm{CI}$

\begin{tabular}{lcccccc} 
Metabolite & AUC & Standard error & Lower & Upper & Sensitivity & Specificity \\
\hline Phosphorylcholine & 0.913 & 0.035 & 0.843 & 0.982 & 0.854 & 1.000 \\
L-Acetylcarnitine & 0.803 & 0.054 & 0.698 & 0.907 & 0.762 & 0.708 \\
Arachidonic acid & 0.887 & 0.050 & 0.789 & 0.985 & 0.905 & 0.833 \\
Glycocholic acid $_{\text {Four metabolites model }}{ }^{\mathrm{a}}$ & 0.808 & 0.056 & 0.697 & 0.918 & 0.729 & 0.810 \\
\hline
\end{tabular}

${ }^{a}$ The four metabolites model comprised phosphorylcholine, L-acetylcarnitine, arachidonic acid and glycocholic acid. CI, confidence interval; AUC, area under the curve.

A

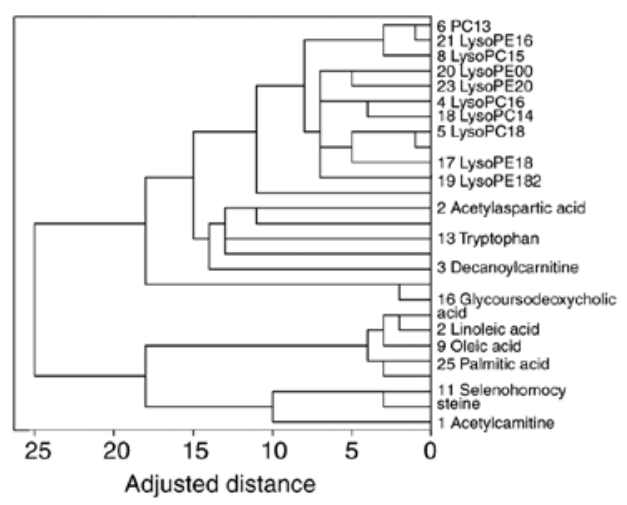

C

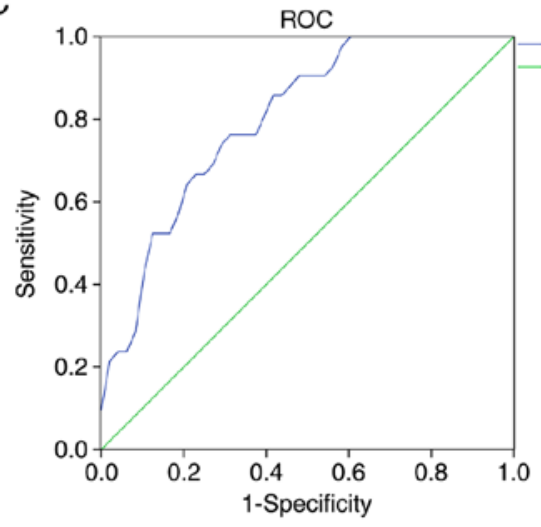

E

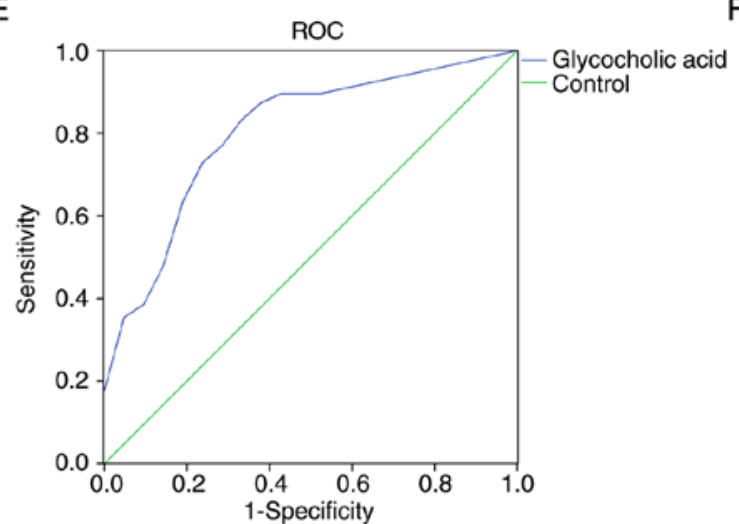

B

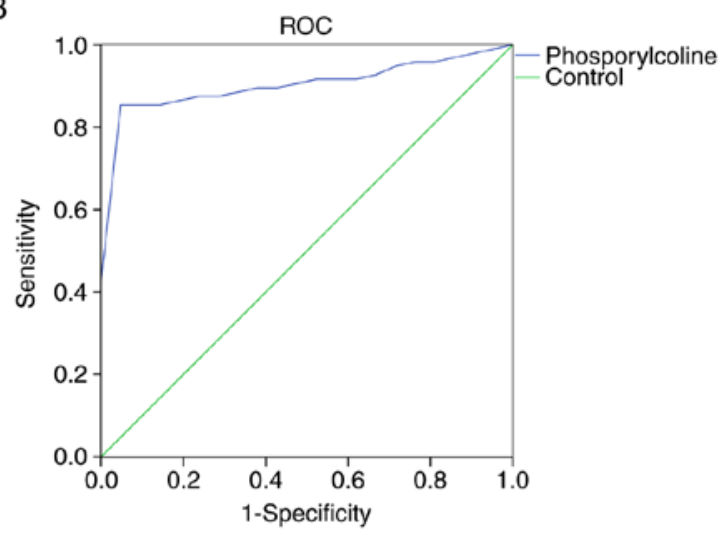

D

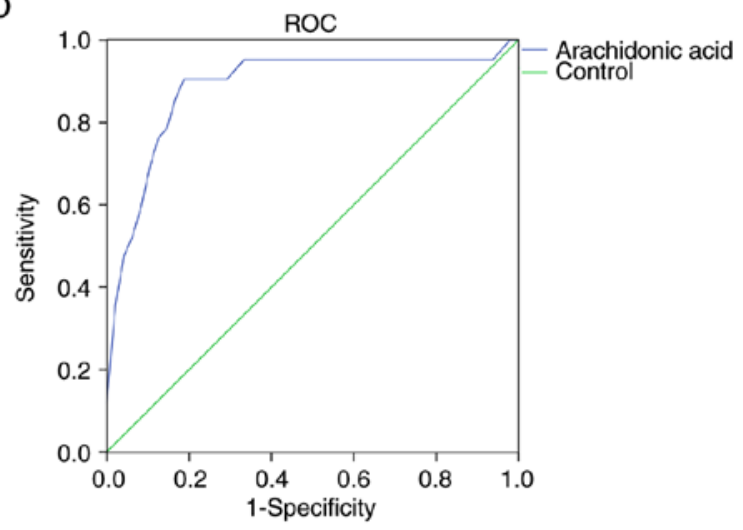

$\mathrm{F}$

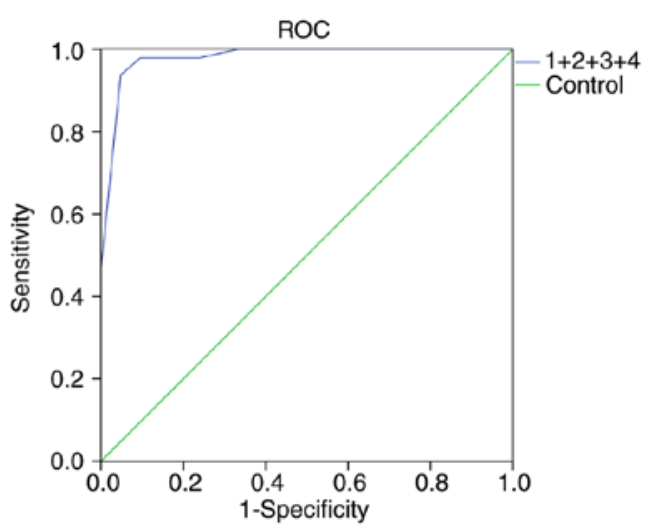

Figure 3. Results of cluster and ROC curve analysis. (A) Results of cluster analysis of 27 potential biomarkers. ROC of (B) L-acetylcarnitine, (C) phosphorylcholine, (D) glycocholic acid and (E) arachidonic acid. (F) ROC of the combined model (1, phosphorylcholine; 2, L-acetylcarnitine; 3 , arachidonic acid; 4, glycocholic acid). ROC, receiver operating characteristic. 


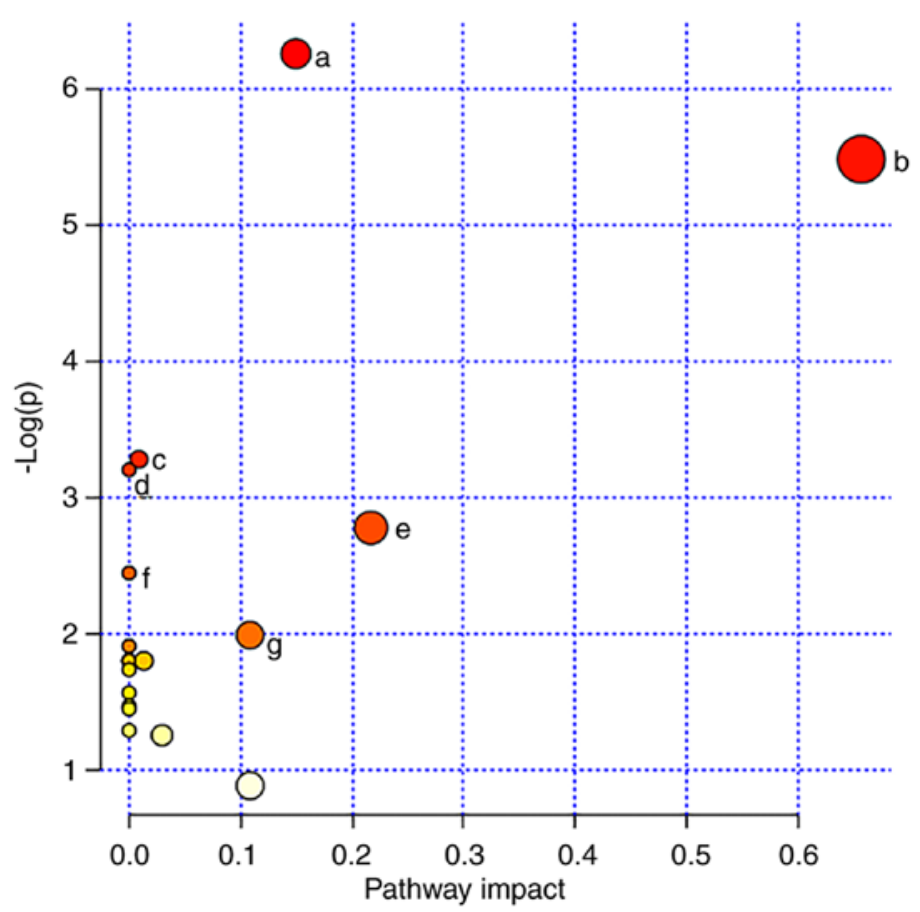

Figure 4. Analysis of gastric cardia adenocarcinoma-associated metabolic pathways. a, 'Glycerophospholipid metabolism'; b, 'linoleic acid metabolism'; c, 'primary bile acid biosynthesis'; d, 'fatty acid biosynthesis'; e, 'arachidonic acid metabolism'; f, 'aminoacyl-tRNA biosynthesis'; g, 'seleno amino acid metabolism'. A circle of darker color indicates a greater $-\log (\mathrm{p})$ value, and a circle of larger size indicates a greater pathway impact value.

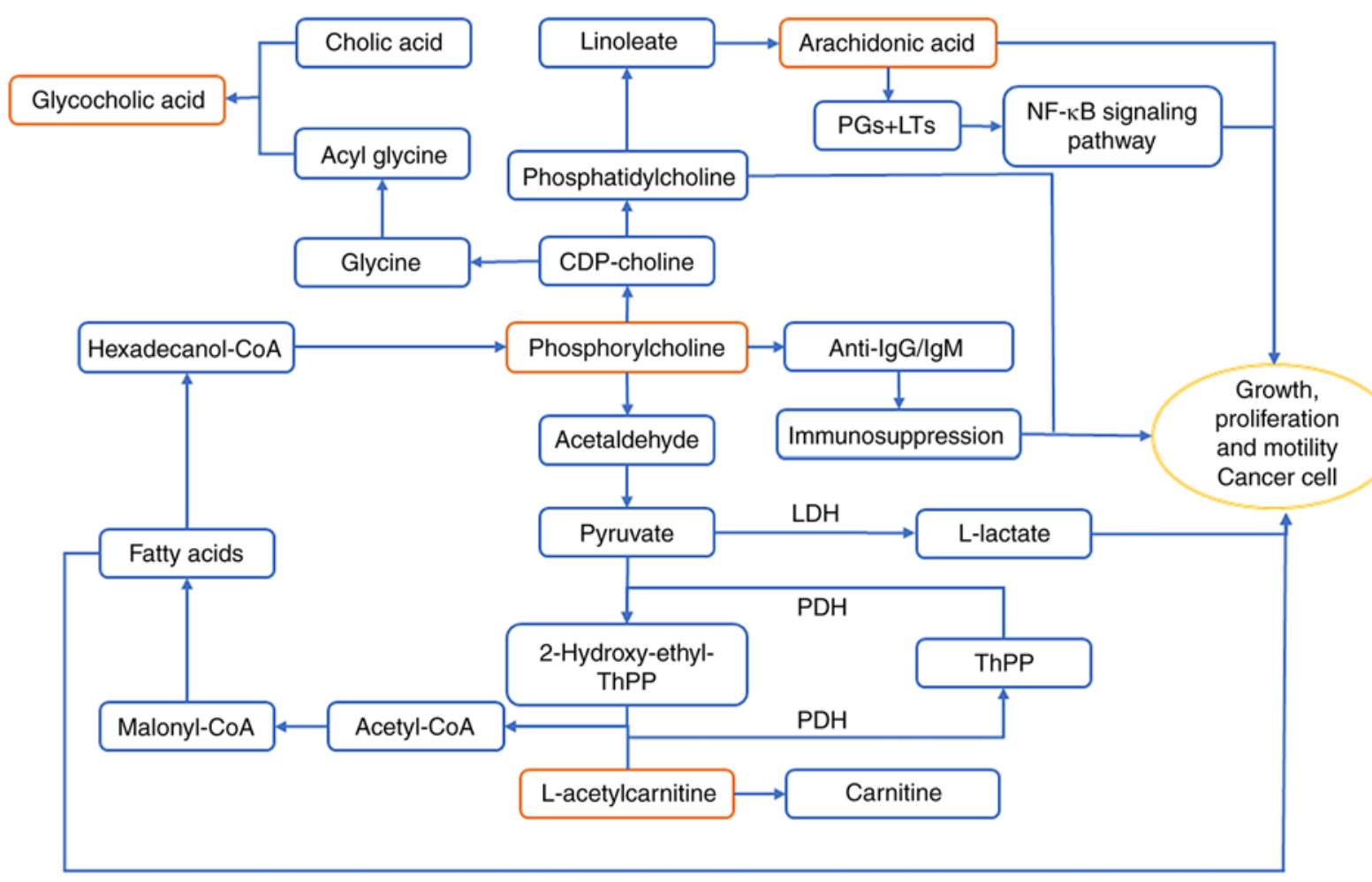

Figure 5. Schematic diagram of the metabolism of phosphorylcholine, L-acetylcarnitine, arachidonic acid and glycocholic acid.

The present study used UPLC-Q/TOF MS plasma metabolomics analysis to determine the characteristic metabolic fingerprints in the plasma of patients with GCA. An OPLS-DA model based on plasma metabolomics data exhibited sufficient sensitivity and specificity to distinguish between patients with
GCA and healthy controls. Using the cut-off criteria of VIP $>1$ and $\mathrm{P}<0.05,27$ potential biomarkers for the diagnosis of GCA were identified. Systematic cluster analysis and area under ROC curve values were used to establish a combined diagnostic model consisting of phosphorylcholine, glycocholic acid, arachidonic 
acid and L-acetylcarnitine for the early diagnosis of GCA. Compared with a single indicator diagnostic model, the combined diagnostic model exhibited significantly improved sensitivity and specificity for the diagnosis of GCA. Additionally, the four key biomarkers and associated metabolic pathways were investigated to shed new light on the pathogenesis of GCA.

L-acetylcarnitine serves as an acetyl carrier and provides an acetyl group for coenzyme A (CoA) (40). Acetyl-CoA is required for the de novo synthesis of fatty acids (41). Hexadecanol-CoA, a product of fatty acid metabolism, is metabolized to produce phosphorylcholine and glycerophospholipid. Acetaldehyde, which is produced from phosphorylcholine, is the link between the glycerophospholipid and pyruvate metabolic pathways (42). Additionally, acetyl-CoA synthesis is catalyzed by pyruvate dehydrogenase (43). Increased L-acetylcarnitine levels in patients with GCA are associated with excessive fatty acids and previous studies have revealed that fatty acids, particularly unsaturated fatty acids, are a major source of energy for cancer cells $(44,45)$.

Previous studies have demonstrated that phosphorylcholine metabolism is closely associated with the immune response $(46,47)$. Phosphorylcholine is taken up by lymphoid B cells, which produce anti-phosphorylcholine immunoglobulin $\mathrm{G}$ and $\mathrm{M}$ antibodies that target cancer cells (48). However, in the present study, the phosphorylcholine levels in patients with GCA were significantly reduced compared with healthy subjects, suggesting a weakened immune response. Phosphorylcholine synthesizes cytidine 5'-diphosphocholine, a reaction catalyzed by phosphate cytidylyltransferase 1 (49). Phosphatidylcholine is subsequently produced by ethanolamine phosphotransferase 1 or choline phosphotransferase 1 (50). Phosphatidylcholine promotes apoptosis in the human gastric cancer cell line BGC823, downregulates the expression of ATP binding cassette subfamily $\mathrm{F}$ member 2 and reduces the number of cancer stem cells $(51,52)$. Therefore, decreased phosphorylcholine levels in patients with GCA may provide a favorable environment for the proliferation of GCA cells.

Phosphatidylcholine is used to synthesize linoleic acid through the linoleic acid metabolic pathway, which can be further used in the synthesis of arachidonic acid (53). The present study revealed that the levels of arachidonic acid in patients with GCA were significantly increased compared with healthy subjects. Eicosanoids, including prostaglandins (PGs) and leukotrienes (LTs) are synthesized by cyclooxygenase-2 (COX-2) and 5-lipoxygenase (5-LOX) from arachidonic acid (54). In turn, PGs and LTs upregulate the expression of COX-2 and 5-LOX via a positive feedback amplification mechanism $(55,56)$. PGs and LTs promote cancer cell proliferation and tumor growth by activating the mitogen-activated protein kinase/nuclear factor- $\kappa \mathrm{B}$ signaling pathway $(57,58)$. In addition, $\mathrm{PGs}$ and LTs increase the survival, proliferation, invasion and migration and decrease apoptosis of tumor epithelial cells by regulating multiple signaling pathways (56), such as protein kinase C/extracellular signal regulated kinase pathway and Ras-Raf-Erk pathway $(59,60)$. Therefore, the arachidonic acid content in patients with GCA may increase to allow the proliferation of tumor cells.

Bile acids, particularly unconjugated bile acids, are implicated in gastroduodenal reflux (61). Long-term gastroduodenal reflux may result in chronic inflammation (62), which may lead to muscle atrophy in the stomach tissue, intestinal erosion and the development of cancer (63). The accumulation of bile salts can contribute towards progressive liver damage and fibrosis; therefore, the levels of bile acids in the blood and tissues are highly regulated (64). Glycocholic acid, which is a combination of acetyl glycine (produced by cytidine 5'-diphosphocholine) and bile acid, serves as a detergent and promotes the absorption of fat as well as its own absorption, thereby preventing cholestasis (65). The present study revealed a significant decrease in glycocholic acid levels in patients with GCA compared with healthy subjects, consistent with the aforementioned studies.

A large sample size is often required for clinical research due to individual variations. However, the present study implemented strict inclusion/exclusion criteria and required informed consent for each patient, which resulted in only 21 patients being available for sampling. One limitation of this study is the low number of samples, which does not allow further analysis, for example, to identify the biomarkers for TNM staging of GCA, which would require a large number of additional clinical samples. Future studies will continue to collect clinical samples and perform in-depth analysis. Additionally, digestive tract diseases are closely associated with imbalances in the intestinal flora (66). As a limited number of studies have investigated the association between GCA and the intestinal flora, future studies are warranted. The multi-omics fusion of metabolomics, proteomics, genomics and metagenomics may aid the optimization of the early diagnostic model of GCA described in the current study and elucidate the underlying mechanisms of the disease. The present study identified four biomarkers and established a combined diagnostic model for early diagnosis of GCA, which may achieve progress in the prevention and treatment of GCA. Further metabolic pathway analysis is required to better understand the molecular pathogenesis of GCA.

\section{Acknowledgements}

Not applicable.

\section{Funding}

The present study was supported by the Hangzhou Science and Technology Commission (grant no. 20140633B41) and the Medical Innovation Project of PLA Nanjing Military Area Command (grant no. 2013MS150).

\section{Availability of data and materials}

The datasets used and/or analyzed during the present study are available from the corresponding author on reasonable request.

\section{Authors' contributions}

YS analyzed the data and wrote the manuscript. SL analyzed the data and edited the manuscript. JL acquired and analyzed the data. XX analyzed the data and edited the manuscript. $\mathrm{ZH}$ collected the clinical samples and acquired the data. XW designed the study. SY designed the study and reviewed and edited the manuscript. All authors read and approved the final version of the manuscript. 


\section{Ethics approval and consent to participate}

The present study was approved by Institutional Review Board of the 903rd Hospital of PLA (approval no. 20140501; Hangzhou, China) and Institutional Review Board of People's Hospital of Yangzhong City (approval no. IRB201404; Yangzhong, China). Written informed consent was obtained from all participants.

\section{Patient consent for publication}

Not applicable.

\section{Competing interests}

The authors declare that they have no competing interests.

\section{References}

1. Colquhoun A, Arnold M, Ferlay J, Goodman KJ, Forman D and Soerjomataram I: Global patterns of cardia and non-cardia gastric cancer incidence in 2012. Gut 64: 1881-1888, 2015.

2. Wang LD, Zheng S, Zheng ZY and Casson AG: Primary adenocarcinomas of lower esophagus, esophagogastric junction and gastric cardia: In special reference to China. World J Gastroenterol 9: 1156-1164, 2003.

3. Huang Q, Li R, Xu GF, Zhou D, Fan XS and Zou XP: Emerging evidence supports grouping by location of early gastric carcinoma for appropriate clinical management in Chinese patients. J Dig Dis 19: 730-736, 2018.

4. Abdi E, Latifi-Navid S, Zahri S, Yazdanbod A and Pourfarzi F: Risk factors predisposing to cardia gastric adenocarcinoma: Insights and new perspectives. Cancer Med 8: 6114-6126, 2019.

5. Ze-Long Y, Guo-Hui M, Lin Z, Wei-Hong Y, Ke-Cheng Z and Yan-Wen J: Survival trends of patients with surgically resected gastric cardia cancer from 1988 to 2015 a population-based study in the United States. Am J Clin Oncol 42: 581-587, 2019.

6. Kutup A, Yekebas EF and Izbicki JR: Current diagnosis and future impact of micrometastases for therapeutic strategies in adenocarcinoma of the esophagus, gastric cardia, and upper gastric third. Recent Results Cancer Res 182: 115-125, 2010.

7. An JY, Baik YH, Choi MG, Noh JH, Sohn TS, Bae JM and Kim S: The prognosis of gastric cardia cancer after R0 resection. Am J Surg 199: 725-729, 2010.

8. Nishimata H, Maruyama M, Shimaoka S, Nishimata Y, Ohi H, Niihara T, Nioh T, Matsuda A, Tashiro K and Torimaru H: Early gastric carcinomas in the cardiac region: Diagnosis with double-contrast $\mathrm{x}$-ray studies. Abdom Imaging 28: 486-491, 2003.

9. Togo R, Yamamichi N, Mabe K, Takahashi Y, Takeuchi C, Kato M, Sakamoto N, Ishihara K, Ogawa T and Haseyama M Detection of gastritis by a deep convolutional neural network from double-contrast upper gastrointestinal barium X-ray radiography. J Gastroenterol 54: 321-329, 2019.

10. Carandang N, Schuman BM and Priest RJ: The gastrocamera in the diagnosis of stomach disease. JAMA 204: 717-722, 1968.

11. Fujiyoshi T, Miyahara R, Funasaka K, Furukawa K, Sawada T and Maeda K, Yamamura T, Ishikawa T, Ohno E, Nakamura M, et al: Utility of linked color imaging for endoscopic diagnosis of early gastric cancer. World J Gastroenterol 25: 1248-1258, 2019.

12. Shen L, Zhou C, Liu L, Zhang L, Lu D, Cai J, Zhao L, Chu R, Zhou J and Zhang J: Application of oral contrast trans-abdominal ultrasonography for initial screening of gastric cancer in rural areas of China. Dig Liver Dis 49: 918-923, 2017.

13. Dai CL, Yang ZG, Xue LP and Li YM: Application value of multi-slice spiral computed tomography for imaging determination of metastatic lymph nodes of gastric cancer. World $\mathrm{J}$ Gastroenterol 19: 5732-5737, 2013.

14. Jiang M, Wang X, Shan X, Pan D, Jia Y, Ni E, Hu Y and Huang H: Value of multi-slice spiral computed tomography in the diagnosis of metastatic lymph nodes and $\mathrm{N}$-stage of gastric cancer. J Int Med Res 47: 281-292, 2019.

15. Chan AW, Mercier P, Schiller D, Bailey R, Robbins S, Eurich DT, Sawyer MB and Broadhurst D: (1)H-NMR urinary metabolomic profiling for diagnosis of gastric cancer. Br J Cancer 114: 59-62, 2016.
16. Tatsubayashi T, Tanizawa Y, Miki Y, Tokunaga M, Bando E, Kawamura T, Sugiura T, Kinugasa Y, Uesaka K and Terashima M: Treatment outcomes of hepatectomy for liver metastases of gastric cancer diagnosed using contrast-enhanced magnetic resonance imaging. Gastric Cancer 20: 387-393, 2017.

17. Van Vliet EP, Hermans JJ, De Wever W, Eijkemans MJ, Steyerberg EW, Faasse C, van Helmond EP, de Leeuw AM, Sikkenk AC, de Vries AR, et al: Radiologist experience and $\mathrm{CT}$ examination quality determine metastasis detection in patients with esophageal or gastric cardia cancer. Eur Radiol 18: 2475-2484, 2008.

18. Li L, Pang X, Zhu Z, Lu L Yang J, Cao J and Fei S: GTPBP4 promotes gastric cancer progression via regulating P53 activity. Cell Physiol Biochem 45: 667-676, 2018

19. Zhou F, Xu Y, Shi J, Lan X, Zou X, Wang L and Huang Q: Expression profile of E-cadherin, estrogen receptors, and P53 in early-onset gastric cancers. Cancer Med 5: 3403-3411, 2016.

20. Yin J, Pan H, Long T, Lv L, Zhai P, Liu C, Shao A, Shi Y, Sun Y, Zhu J, et al: Polymorphisms of VDR gene and risk of gastric cardiac adenocarcinoma in Chinese population. Oncotarget 8: 45531-45543, 2017.

21. Zhou RM, Wang N, Chen ZF, Duan YN, Sun DL and Li Y: Polymorphisms in promoter region of FAS and FASL gene and risk of cardia gastric adenocarcinoma. J Gastroenterol Hepatol 25: 555-561, 2010.

22. Zhang J, Cui Y, Kuang G, Li Y, Wang N, Wang R, Guo W, Wen D, Wei L, Yu F and Wang S: Association of the thymidylate synthase polymorphisms with esophageal squamous cell carcinoma and gastric cardiac adenocarcinoma. Carcinogenesis 25 : 2479-2485, 2004.

23. Luo T, Chen W, Wang L and Zhao H: CA125 is a potential biomarker to predict surgically incurable gastric and cardia cancer: A retrospective study. Medicine (Baltimore) 95: e5297, 2016.

24. Williams GH, Swinn R, Prevost AT, de Clive-Lowe P, Halsall I, Going JJ, Hales CN, Stoeber K and Middleton SJ: Diagnosis of oesophageal cancer by detection of minichromosome maintenance 5 protein in gastric aspirates. Br J Cancer 91: 714-719, 2004.

25. Liu M, Li JS, Tian DP, Huang B, Rosqvist S and Su M: MCM2 expression levels predict diagnosis and prognosis in gastric cardiac cancer. Histol Histopathol 28: 481-492, 2013.

26. Cheng Y, Zhang J, Li Y, Wang Y and Gong J: Proteome analysis of human gastric cardia adenocarcinoma by laser capture microdissection. BMC Cancer 7: 191, 2007.

27. Liu HN, Liu TT, Wu H, Chen YJ, Tseng YJ, Yao C, Weng SQ, Dong L and Shen XZ: Serum microRNA signatures and metabolomics have high diagnostic value in colorectal cancer using two novel methods. Cancer Sci 109: 1185-1194, 2018.

28. Zhang A, Sun H, Yan G, Wang P, Han Y and Wang X: Metabolomics in diagnosis and biomarker discovery of colorectal cancer. Cancer Lett 345: 17-20, 2014.

29. Wang X, Zhang A and Sun H: Power of metabolomics in diagnosis and biomarker discovery of hepatocellular carcinoma. Hepatology 57: 2072-2077, 2013.

30. Kobayashi T, Nishiumi S, Ikeda A, Yoshie T, Sakai A, Matsubara A, Izumi Y, Tsumura H, Tsuda M, Nishisaki H, et al: A novel serum metabolomics-based diagnostic approach to pancreatic cancer. Cancer Epidemiol Biomarkers Prev 22: 571-579, 2013.

31. Zhang G, He P, Tan H, Budhu A, Gaedcke J, Ghadimi BM, Ried T, Yfantis HG, Lee DH, Maitra A, et al: Integration of metabolomics and transcriptomics revealed a fatty acid network exerting growth inhibitory effects in human pancreatic cancer. Clin Cancer Res 19: 4983-4993, 2013.

32. Abbassi-Ghadi N, Kumar S, Huang J, Goldin R, Takats Z and Hanna GB: Metabolomic profiling of oesophago-gastric cancer: A systematic review. Eur J Cancer 49: 3625-3637, 2013.

33. Wang X, Yan SK, Dai WX, Liu XR, Zhang WD and Wang JJ: A metabonomic approach to chemosensitivity prediction of cisplatin plus 5-fluorouracil in a human xenograft model of gastric cancer. Int J Cancer 127: 2841-2850, 2010.

34. Cai Z, Zhao JS, Li JJ, Peng DN, Wang XY, Chen TL, Qiu YP, Chen PP, Li WJ, Xu LY, et al: A combined proteomics and metabolomics profiling of gastric cardia cancer reveals characteristic dysregulations in glucose metabolism. Mol Cell Proteomics 9: 2617-2628, 2010.

35. Zhen C: Glucose metabolic pathway in gastric cardia cancer and the role of microcilia in tumorogenesis [Chinese]. Shanghai Institutes for Biological Sciences, 2011.

36. Patriarca S, Ferretti S and Zanetti R: TNM Classification of malignant tumours-Eighth edition: Which news? Epidemiol Prev 41: 140-143, 2017 (In Italian). 
37. Chong J, Soufan O, Li C, Caraus I, Li S, Bourque G, Wishart DS and Xia J: MetaboAnalyst 4.0: towards more transparent and integrative metabolomics analysis. Nucleic Acids Res 46: W486-W494, 2018.

38. Amini N, Spolverato G, Kim Y, Squires MH, Poultsides GA, Fields R, Schmidt C, Weber SM, Votanopoulos K, Maithel SK and Pawlik TM: Clinicopathological features and prognosis of gastric cardia adenocarcinoma: A multi-institutional US study. J Surg Oncol 111: 285-292, 2015.

39. Wang J, Zhang H, Zhou X, Wang T, Zhang J, Zhu W, Zhu H and Cheng W: Five serum-based miRNAs were identified as potential diagnostic biomarkers in gastric cardia adenocarcinoma. Cancer Biomark 23: 193-203, 2018.

40. von Glutz G and Walter P: Compartmentation of acetyl-coA in rat-liver mitochondria. Eur J Biochem 60: 147-152, 1975.

41. Svensson RU, Parker SJ, Eichner LJ, Kolar MJ, Wallace M, Brun SN, Lombardo PS, Van Nostrand JL, Hutchins A, Vera L, et al: Inhibition of acetyl-CoA carboxylase suppresses fatty acid synthesis and tumor growth of non-small-cell lung cancer in preclinical models. Nat Med 22: 1108-1119, 2016.

42. Scholz R, Olson MS, Schwab AJ, Schwabe U, Noell C and Braun W: The effect of fatty acids on the regulation of pyruvate dehydrogenase in perfused rat liver. Eur J Biochem 86: 519-530, 1978.

43. Ke J, Behal RH, Back SL, Nikolau BJ, Wurtele ES and Oliver DJ: The role of pyruvate dehydrogenase and acetyl-coenzyme A synthetase in fatty acid synthesis in developing arabidopsis seeds. Plant Physiol 123: 497-508, 2000.

44. Lucenteforte E, Bosetti C, Gallus S, Bertuccio P, Pelucchi C, Tavani A, La Vecchia C and Negri E: Macronutrients, fatty acids and cholesterol intake and stomach cancer risk. Ann Oncol 20: 1434-1438, 2009.

45. Wen YA, Xing XP, Harris JW, Zaytseva YY, Mitov MI, Napier DL, Weiss HL, Mark Evers B and Gao T: Adipocytes activate mitochondrial fatty acid oxidation and autophagy to promote tumor growth in colon cancer. Cell Death Dis 8: e2593, 2017.

46. Caligiuri G, Khallou-Laschet J, Vandaele M, Gaston AT, Delignat S, Mandet C, Kohler HV, Kaveri SV and Nicoletti A: Phosphorylcholine-targeting immunization reduces atherosclerosis. J Am Coll Cardiol 50: 540-546, 2007.

47. Claflin JL, Lieberman R and Davie JM: Clonal nature of the immune response to phosphorylcholine. I. Specificity, class, and idiotype of phosphorylcholine-binding receptors on lymphoid cells. J Exp Med 139: 58-73, 1974.

48. Sigal NH, Pickard AR, Metcalf ES, Gearhart PJ and Klinman NR: Expression of phosphorylcholine-specific B cells during murine development. J Exp Med 146: 933-948, 1977.

49. Feldman DA, Rounsifer ME and Weinhold PA: The stimulation and binding of CTP: Phosphorylcholine cytidylyltransferase by phosphatidylcholine-oleic acid vesicles. Biochim Biophys Acta 833: 429-437, 1985.

50. Gasull T, Sarri E, DeGregorio-Rocasolano N and Trullas R: NMDA receptor overactivation inhibits phospholipid synthesis by decreasing choline-ethanolamine phosphotransferase activity. J Neurosci 23: 4100-4107, 2003.

51. Mori N, Wildes F, Kakkad S, Jacob D, Solaiyappan M, Glunde K and Bhujwalla ZM: Choline kinase-alpha protein and phosphatidylcholine but not phosphocholine are required for breast cancer cell survival. NMR Biomed 28: 1697-1706, 2015

52. Zhang H, Song H, Yuan R, Zhang X, Yu H, Zhao Y and Jiang T: Polyene phosphatidylcholine overcomes oxaliplatin resistance in human gastric cancer BGC823 cells. Biochem Biophys Res Commun 497: 108-114, 2018.
53. Bereziat G, Chambaz J, Trugnan G, Pepin D and Polonovski J: Turnover of phospholipid linoleic and arachidonic acids in human platelets from plasma lecithins. J Lipid Res 19: 495-500, 1978.

54. Wang D and Dubois RN: Eicosanoids and cancer. Nat Rev Cancer 10: 181-193, 2010.

55. Nieves D and Moreno JJ: Effect of arachidonic and eicosapentaenoic acid metabolism on RAW 264.7 macrophage proliferation. J Cell Physiol 208: 428-434, 2006.

56. Yarla NS, Bishayee A, Sethi G, Reddanna P, Kalle AM, Dhananjaya BL, Dowluru KS, Chintala R and Duddukuri GR: Targeting arachidonic acid pathway by natural products for cancer prevention and therapy. Semin Cancer Biol 40-41: 48-81, 2016.

57. Poligone $\mathrm{B}$ and Baldwin AS: Positive and negative regulation of NF-kappaB by COX-2: roles of different prostaglandins. J Biol Chem 276: 38658-38664, 2001

58. Bortuzzo C, Hanif R, Kashfi K, Staiano-Coico L, Shiff SJ and Rigas B: The effect of leukotrienes B and selected HETEs on the proliferation of colon cancer cells. Biochim Biophys Acta 1300: 240-246, 1996.

59. Yu L, Wu WK, Li ZJ, Li HT, Wu YC and Cho CH: Prostaglandin E-2 promotes cell proliferation via protein kinase C/extracellular signal regulated kinase pathway-dependent induction of c-Myc expression in human esophageal squamous cell carcinoma cells. Int J Cancer 125: 2540-2546, 2009.

60. U1 Islam S, Shehzad A and Lee YS: Prostaglandin E-2 inhibits resveratrol-induced apoptosis through activation of survival signaling pathways in HCT-15 cell lines. Anim Cells Syst 19: 374-384, 2015.

61. Kauer WK, Peters JH, DeMeester TR, Feussner H, Ireland AP, Stein HJ and Siewert RJ: Composition and concentration of bile acid reflux into the esophagus of patients with gastroesophageal reflux disease. Surgery 122: 874-881, 1997.

62. Fitzgerald RC, Abdalla S, Onwuegbusi BA, Sirieix P, Saeed IT and Burnham WR and Farthing MJ: Inflammatory gradient in Barrett's oesophagus: Implications for disease complications. Gut 51: 316-322, 2002

63. Tatsugami M, Ito $M$, Tanaka S, Yoshihara M, Matsui $H$, Haruma $\mathrm{K}$ and Chayama K: Bile acid promotes intestinal metaplasia and gastric carcinogenesis. Cancer Epidemiol Biomarkers Prev 21: 2101-2107, 2012.

64. Messner CJ, Mauch L and Suter-Dick L: Bile salts regulate CYP7A1 expression and elicit a fibrotic response and abnormal lipid production in 3D liver microtissues. Toxicol In Vitro 60: 261-271, 2019.

65. Heubi JE, Setchell KD, Jha P, Buckley D, Zhang WJ, Rosenthal P, Potter C, Horslen S and Suskind D: Treatment of bile acid amidation defects with glycocholic acid. Hepatology 61: 268-274, 2015

66. Abreu MT and Peek RM Jr: Gastrointestinal malignancy and the microbiome. Gastroenterology 146: 1534-1166, 2014.

67. Hu H, Krasinskas A and Willis J: Perspectives on current tumor-node-metastasis (TNM) staging of cancers of the colon and rectum. Semin Oncol 38: 500-510, 2011.

This work is licensed under a Creative Commons Attribution-NonCommercial-NoDerivatives 4.0 International (CC BY-NC-ND 4.0) License. 